\title{
The relationship between blood sugar levels (glycosylated haemoglobin) and the risk of development of diabetic retinopathy
}

\author{
Priyadharshini N. ${ }^{1}$, Annamalai R. ${ }^{2}$, Muthayya M.K. ${ }^{3}$ \\ ${ }^{1}$ Dr. N. Priyadharshini, Post Graduate, Ophthalmology, ${ }^{2}$ Dr. Radha Annamalai, Professor, Ophthalmology, \\ ${ }^{3}$ Dr. Muthayya Muthukumar, Professor, Ophthalmology, all authors are affiliated with Sri Ramachandra University, \\ Porur, Chennai, Tamilnadu, India.
}

Address for Correspondence: Dr. Radha Annamalai, Professor of Ophthalmology, Sri Ramachandra University, Porur, Chennai, India. E-mail: drradhaannamalai@yahoo.co.in

\begin{abstract}
Introduction: Diabetic retinopathy is a major cause of visual impairment and blindness in India and with its early detection and timely treatment, the risk of visual loss can be reduced significantly. Onset and progression of retinopathy is determined by fasting and postprandial blood sugar levels and other risk factors such as duration of diabetes, hypertension, nephropathy, hypercholesterolemia and obesity. This study was done to evaluate the role of glycosylated hemoglobin and its association with the severity of retinopathy. Materials and Methods: A total of 100 patients were included. This was a prospective study conducted over 1 year in Sri Ramachandra University, Chennai, India. Fundus examination was done using slit lamp biomicroscopy and indirect ophthalmoscopy on all patients. Ancillary investigations such as fundus fluorescein angiography and optical coherence tomography were performed. HbA1c was measured along with fasting, postprandial, lipid profile and urine sugars. Results: High and uncontrolled levels of HbA1c were associated with maculopathy. A statistically significant difference $(\mathrm{p}<0.01)$ was found in them. The retinopathy however was not related to $\mathrm{HbA1C}$ alone. The most frequent type of maculopathy noted was cystoid macular oedema and the level above which it occurred was $7 \%$ of HbA1C. Besides HbA1C, the other important factor that was associated with maculopathy was duration of diabetes mellitus. Conclusion: HbA1c value $>7.0 \%$ was significantly related with maculopathy. The severity of retinopathy is dependent on the level of blood sugar and duration of diabetes. Control of sugar levels is crucial to prevent diabetic retinopathy and its complications which can lead to irreversible complications in the eye.
\end{abstract}

Keywords: Diabetes mellitus, Retinopathy, Glycosylated haemoglobin, Maculopathy, Indirect ophthalmoscopy, Slit lamp biomicroscopy

\section{Introduction}

The prevalence of diabetes mellitus for all age-groups worldwide was estimated to be $2.8 \%$ in 2000 and $4.4 \%$ in 2030. The total number of people with diabetes is projected to rise from 171 million in 2000 to 366 million in 2030. Diabetic retinopathy remains a major cause of visual impairment and blindness in India and with its early detection and timely treatment, the risk of visual loss can be reduced significantly. The evidence that screening for diabetic retinopathy is cost effective has led to developments, over the past 20

Manuscript received: $2^{\text {nd }}$ January 2017

Reviewed: $10^{\text {th }}$ January 2017

Author Corrected: $16^{\text {th }}$ January 2017

Accepted for Publication: 24 $4^{\text {th }}$ January 2017 years, of several screening programmes at local, regional, and national levels throughout India and elsewhere, depending on the infrastructure and facilities available.

Various methods have been used to screen for diabetic retinopathy, including ophthalmoscopy (direct and indirect) [1], combining ophthalmoscopy with retinal photography by fundus camera, fundus fluorescein angiography and optical coherence tomography. India is already being termed as the "Diabetic capital of the world", with the number of patients with diabetes expected to rise from 40.9 million, at present, to 60.9 
million by 2025 [2]. Glycosylated haemoglobin HbA1c has been used as a predictor of the diabetic status and also to assess the control of diabetes mellitus during routine evaluation of these patients. The Early Treatment Diabetic Retinopathy Study (EDTRS) has identified criteria for diagnosis and staging of diabetic retinopathy [3]. Improvement of diabetic status has long term benefits in control of diabetic retinopathy. This has been studied by Diabetes Control and Complications Trial (DCCT), the Stockholm Interventional Study, and the United Kingdom Prospective Diabetes Study (UKPDS) [4]. The role of HbA1C with the occurrence of different stages of retinopathy have been reported but with no conclusive data. The association between $\mathrm{HbA} 1 \mathrm{c}$ and the occurrence of complications and visual loss, which includes severe NPDR or PDR or clinically significant macular oedema has not been evaluated and presented. We report the influence of $\mathrm{HbA1c}$ on the occurrence of the type of retinopathy or maculopathy in a study conducted in a tertiary eye care hospital in Chennai, India.

The pathogenesis of diabetic retinopathy is based on retinal micro-vascular changes which result in the development of non-proliferative or proliferative retinopathy with or without diabetic maculopathy. Advanced glycation end products (including glycosylated hemoglobin, HbA1c) are known to produce micro-vascular complications in diabetic retinopathy [5]. The severity and the duration of the inadequate glycemic control have been seen to be correlated with a higher risk of increased severity of retinopathy, from non-proliferative to proliferative diabetic retinopathy [6]. All previous studies have explored the association of fasting and post prandial sugars with the occurrence of the different stages of non-proliferative DR (NPDR) and proliferative DR (PDR) [7]. But the causal relationship between HbA1c and the occurrence of visual loss, which includes severe NPDR or PDR or diabetic macular oedema (an important cause of visual impairment in subjects with type II diabetes), has not been done in population-based studies.

\section{Material and Methods}

Sampling and statistical methods, inclusion and exclusion criteria- This was a cohort study done at Sri Ramachandra University, Chennai, Tamil Nadu, India, after obtaining ethics committee approval over a one year period. This was performed in concurrence with the departments of diabetology and internal medicine. The study was approved by the Institutional Ethics Committee, and a written informed consent was obtained from the subjects. During each follow up visit patients underwent evaluation at both the diabetology and ophthalmology clinic. To summarize, 100 persons from the general population with findings of diabetic retinopathy were enumerated based on a multistage random sampling, which was stratified based on the level of $\mathrm{HbA} 1 \mathrm{C}$.

All patients with diabetes underwent a detailed anterior segment and fundus examination. Inclusion criteria were all known diabetes mellitus patients as diagnosed by a physician or on treatment with oral hypoglycemics or insulin injection or asymptomatic individuals, whose fasting blood and those with Hb1c levels showed evidence of diabetes mellitus. Patients with other associated morbidities such as hypertension were excluded from the study.

Materials and Methods used- A fasting blood sugar test was repeated for all those who were classified as provisional diabetics at the other hospitals. All patients whose blood sugar levels were elevated were screened. A written informed consent was obtained from each individual prior to their participation. Diabetic retinopathy was diagnosed based on the Modified Early Treatment Diabetic Retinopathy Study [8]. A detailed medical and ophthalmic history, including the duration of diabetes, the treatment being taken, and other systemic illnesses, were taken. Opinions were obtained from the neurologist, nephrologist and other specialists whenever multisystem involvement of diabetes mellitus was noted.

A detailed ophthalmic examination of both eyes, including the best corrected visual acuity, pachymetry, specular microscopy, slit-lamp anterior segment examination, gonioscopy, intra-ocular pressure measurement by applanation tonometry. Fundus examination was done with indirect ophthalmoscopy and slit lamp biomicroscopy.

Fundus photography, fundus fluorescein angiography and optical coherence tomography was carried out for all patients during the first visit and review. Biochemical investigations included the total serum cholesterol (TC), high density lipoproteins (HDL), lowdensity lipoproteins (LDL), serum triglycerides (TG), 
hemoglobin $(\mathrm{Hb}), \mathrm{HbAlc}$, fasting and postprandial blood sugars and urine for sugar, micro- and macroalbuminuria. HbA1c was measured by the liquid chromatography technique. The data entry was validated and crosschecked, and the statistical analysis was performed. Statistical analyses were performed using SPSS for Windows version 14.0 software (SPSS Inc., Chicago, IL, USA). The level of significance was analysed, in more than two variables, using chi-square trend $\mathrm{p}$ value. A $\mathrm{p}$ value of less than 0.05 was considered significant.

\section{Results}

Among the study population of 500 patients with diabetes mellitus, those with retinopathy were found to be $63 \%$. Individuals with diabetes mellitus were classified as those with retinopathy, maculopathy or both. The HbA1c value for severe NPDR was 8.1(2.1\%), PDR was 7.5(2.4\%) and CSME was 10(2.3\%) (p=0.03). The group with retinopathy included subjects with severe NPDR $(n=18)$, PDR $(n=22)$. Maculopathy included ischemic maculopathy $(n=3)$ and CSME $(n=16)$. A statistically significant difference $(\mathrm{p}<0.03)$ was noted in those with clinically significant macular oedema and HbA1c. The average HbA1c value for severe NPDR was 6.5, PDR was 7and CSME was 9 and above. Also, the duration of DM and recently diagnosed diabetes mellitus were found to be significantly elevated in the maculopathy group as compared to the retinopathy group.

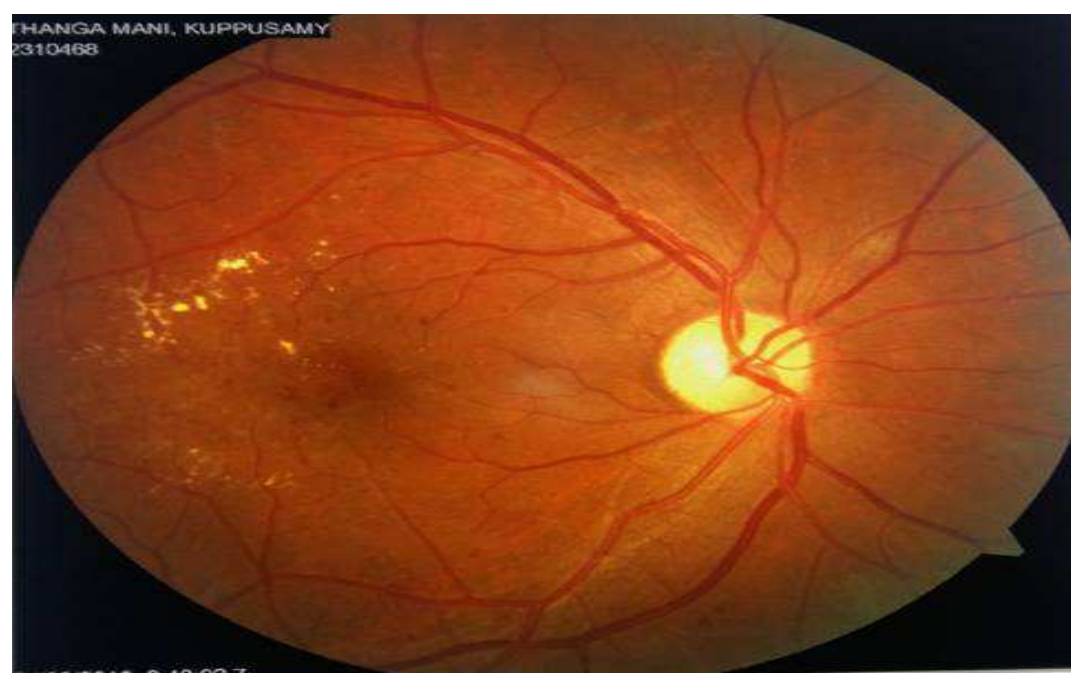

Figure-1: Right eye: Clinically significant macular edema

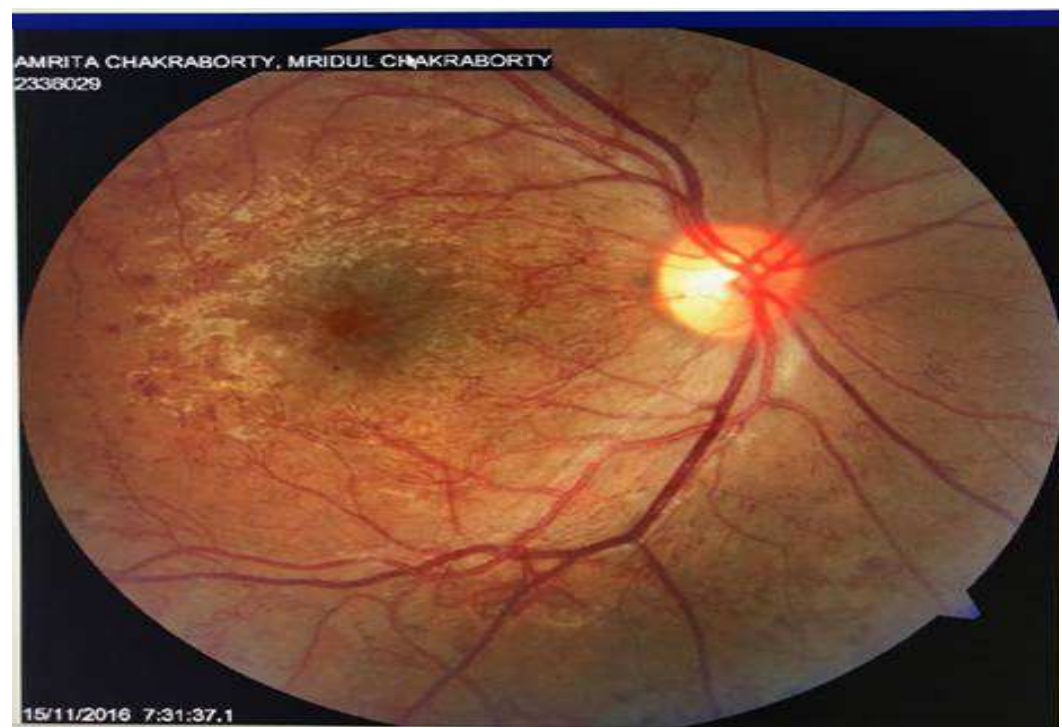

Figure-2: Right eye: Proliferative diabetic retinopathy 


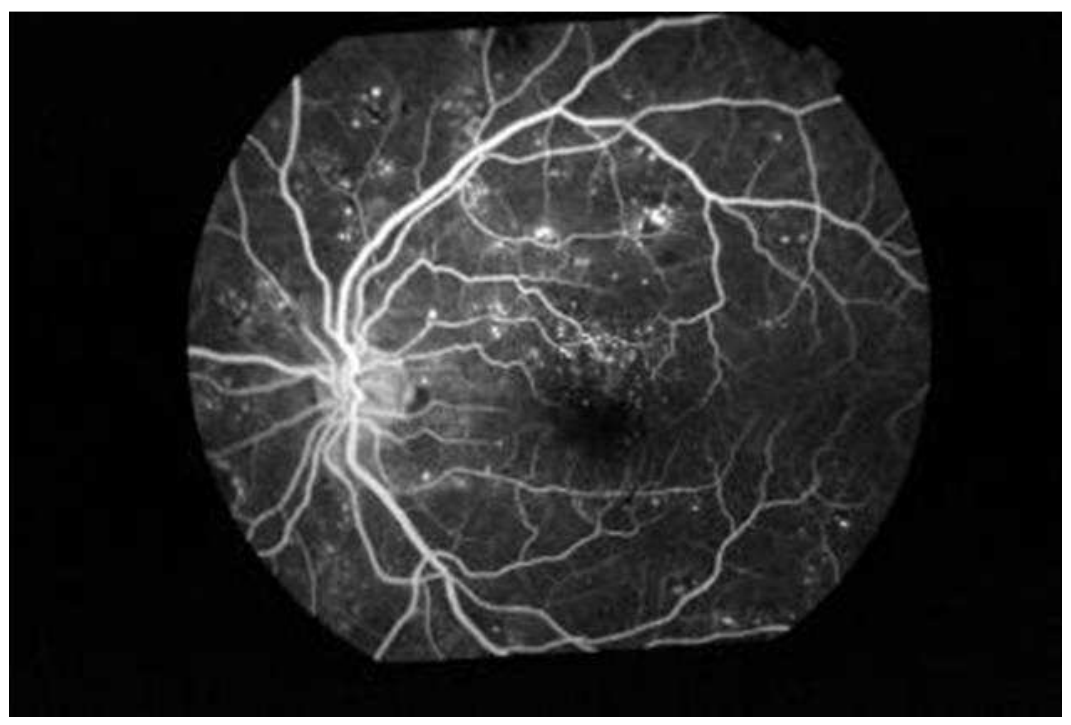

Figure-3: Left eye: Fundus fluorescein angiography shows pinpoint hyperfluorescence suggestive of leaking microaneurysms
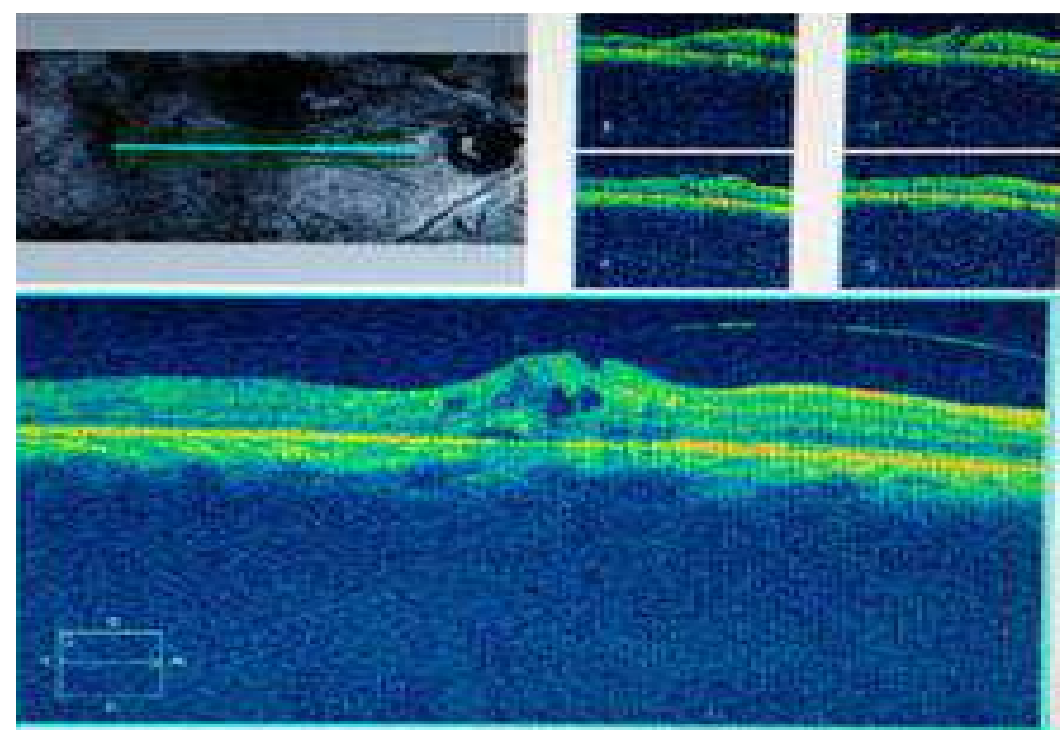

Figure-4: Optical coherence tomography showing cystoid macular edema due to diabetes mellitus

HbA1c was found to be significantly associated with maculopathy (clinically significant macular oedema with $\mathrm{p}=0.003$ ). Distribution based on duration was found to be $45 \%$ in those with disease greater than 15 years, $40 \%$ with disease greater than 10 years and $15 \%$ with disease less than 10 years. When the subgroups with maculopathy were analysed, a statistical significant difference was noted with $\mathrm{p}=0.03$ in those with high Hba1c levels but no difference was noted in terms of duration of the diabetes mellitus and the age groups affected. After follow up for one year of all the patients, the most frequent sight threatening complication was found to be tractional retinal detachment seen in $7 \%$ of patients and vitreous haemorrhage in $2 \%$ of the study group.

On final analysis, our study showed male sex, longer duration of diabetes, poor glycemic control, high HbA1c levels, associated hypertension and nephropathy as the important risk factors for the progression of diabetic retinopathy. Patients aged between $50-60$ years constituted $38 \%$ compared to $28 \%$ of $40-50$ year and only $8 \%$ in extreme age group. Males constituted $68 \%$ compared to $32 \%$ female population studied. Duration of diabetes for 5 to 10 years was $54 \%$ compared to $10 \%$ of more than 15 year of diabetes. Microalbuminuria was present in $22 \%$ patients compared to $78 \%$ with no albuminuria. 
Table-1: HbA1c levels and number of patients

\begin{tabular}{|c|c|c|}
\hline HbA1c & No. of patients & Percentage \\
\hline Less than 8 & 280 & $56 \%$ \\
\hline 8 to 10 & 100 & $20 \%$ \\
\hline More than 8 & 120 & $24 \%$ \\
\hline
\end{tabular}

Table-2: Correlation of incidence of retinopathy / maculopathy

\begin{tabular}{|c|c|c|}
\hline Retinopathy / maculopathy & No. of patients & HbA1c \\
\hline NPDR & $2.1 \%$ & 8.1 \\
\hline PDR & $2.4 \%$ & 7.5 \\
\hline Maculopathy & $2.3 \%$ & 10 \\
\hline
\end{tabular}

\section{Discussion}

The inclusion of HbAlc in the screening protocols has gained significant importance, not only in the diagnosis and management of DM, but also in the onset and progression of DR [9]. It has been seen that compared to the measurement of glucose levels, HbA1c assay is at least as good in defining the level of hyperglycemia at which the prevalence of DR increases [10], [11].

We evaluated the association of $\mathrm{HbAlc}$ with the occurrence of maculopathy and retinopathy in a population based study. Previous HbA1c studies have found $\mathrm{HbA} 1 \mathrm{c}$ to be associated with an increase in the severity of diabetic retinopathy from non-proliferative to proliferative retinopathy but not with maculopaathy. It is important for the clinician to find an association with maculopathy rather than the stage of retinopathy, as even the early stages of macular oedema, without significant retinopathy can cause visual loss. Those with maculopathy had higher HBA1Clevels than the population with only retinopathy.

The duration of diabetic nephropathy was more in those with retinopathy than the retinopathy patients. Previous epidemiological studies have found similar risk factors like duration of diabetes, higher $\mathrm{HbAlc}$ and higher blood pressure independently for NPDR, macular edema and PDR [12]. Glycation of tissue proteins is a well-known pathophysiological mechanism in the complications related to diabetes leading to the formation of advanced glycation end products and HbA1c. Strict glycemic control, when measured by several factors, is strongly associated with a decreased prevalence of micro-vascular complications related to DM like retinopathy. Anitha et al. also found an association of advanced glycation index (AGI) with the severity of DR [12]. We found a strong association of
HbA1c with the development of any type of retinopathy more with maculopathy. Researchers have conclusively proven, in the past, that once the retinopathy develops, there is no relationship between the level of glycemic control and the progression of DR, although the incidence of DR is significantly affected. Earlier reports showed that the prevalence of DR significantly increased at $\mathrm{HbA1c}$ value between $6.0 \%$ and $7.0 \%$. Later, a value of $6.5 \%$ was seen to be a cut-off which could detect at least moderate retinopathy. HbA1c of $6.5 \%$ has now been seen as sufficiently sensitive and specific to identify individuals who are at risk of developing DR.

However, it has still not proven to be an absolute cut-off threshold between normal glycemia and diabetes. On detection of complications, prompt intravitreal injections with steroid or anti vascular endothelial growth factor have to be done. Surgical intervention will be required when there is still visual potential and complications such as vitreous haemorrhage or retinal detachment and the vision can be restored in some patients [13].

Other risk factors such as hypertension, hypercholesterolemia, nephropathy and obesity in patients with diabetic retinopathy can worsen the state of the eye. The prevention of microvascular disease by addressing all the risk factors can play a big role in prevention of further progression [14]. In children, frequent screening should be recommended for neuropathy related complications such as dry eye syndrome, glaucoma and diabetic retinopathy in Type 1 diabetes mellitus. This is because a longer duration of diabetes can cause microvascular involvement and long term follow up is also required [15]. Inflammation can 
be a precursor in diabetes thus mediating negative influences to the neurological and vascular components of the retina. Chronic inflammation can cause changes at the biochemical and molecular level ending in retinopathy and vision loss in these patients [16]. A derangement of serum cholesterol, triglycerides, high density lipoproteins and low density lipoproteins was seen in patients with retinopathy as compared to those without diabetic retinopathy. This has been reported following a study of lipid profile and its correlation with retinopathy in Pakistani population [17]. Fluctuations in the plasma sugar levels have been shown to cause progression of diabetic retinopathy more than nephropathy in patients with diabetes mellitus [18].

This again reinforces the utility of monitoring sugars and their levels over a period of time to detect if there is consistency in sugar control as it affects retinopathy development. The findings of our study, probably, have beneficial aspects in targeted diabetic retinopathy screening programmes. Targeted screening for retinopathy in subjects with diabetes with $\mathrm{HbA1c}>8 \%$ would give maximum yield of sight threatening retinopathies. Regular ophthalmological examinations with methodical documentation during each visit with examination, intraocular pressure measurements, fundus photography and other ancillary investigations such as fundus fluorescein angiography need to be performed in order to prevent blinding complications of diabetic retinopathy.

\section{Conclusion}

Diabetic retinopathy progresses with the duration of disease and the cause of defective vision is usually maculopathy or proliferative retinopathy. Regular ophthalmoscopic examinations are essential in detecting the progression of retinopathy and development of disease characteristics which indicate a need for treatment. Laboratory and clinical experience should be combined in the assessment and the importance of rigid control of $\mathrm{HbA1C}$ in preventing diabetic retinopathy has to be stressed. A major contributor to poor compliance in maintaining HBA1C levels is lack of awareness in patients. In our study we concluded that there was relatively more awareness in younger age groups. Those with multi organ disease due to diabetes mellitus and those under the care of an endocrinologist also showed sharper control.

Funding: Nil, Conflict of interest: None. Permission of IRB: Yes

\section{References}

1. Krishnamurti U, Steffes MW. Glycohemoglobin: a primary predictor of the development or reversal of complications of diabetes mellitus. Clin Chem. 2001;47 (7):1157-65.

2. Seema Abhjeet Kaveeshwar, John Cornwall. The current state of diabetes mellitus in India. Australas Med J. 2014; 7(1): 45-48. DOI: 10.4066/AMJ. 2013.1979

3. Davis MD, Fisher MR, Gangnon RE, Barton F, Aiello LM, Chew EY, Ferris FL 3rd, Knatterud GL. Risk factors for high-risk proliferative diabetic retinopathy and severe visual loss: Early Treatment Diabetic Retinopathy Study Report \#18. Invest Ophthalmol Vis Sci. 1998 Feb;39(2):233-52.

4. Klein R. The Diabetes Control and Complications Trial. Kertes C,ed. Clinical Trials in Ophthalmology: A Summary and Practice Guide. 1998.49-70.

5. Ciulla TA, Amador AG, Zinman B. Diabetic retinopathy and diabetic macular edema: pathophysiology, screening, and novel therapies. Diabetes Care. 2003 Sep;26(9):2653-64.

6. Klein R, Klein BEK, Moss SE, Davis MD, DeMets DL. Glycosylated haemoglobin predicts the incidence and progression of diabetic retinopathy. JAMA 1988 Nov; 260 (19): 2864-71.

7. Lachin JM, Genuth S, Nathan DM, Zinman B, Rutledge BN; DCCT/EDIC Research Group. Effect of glycemic exposure on the risk of microvascular complications in the diabetes control and complications trial--revisited. Diabetes. 2008 Apr; 57 (4):995-1001. doi: 10.2337/db07-1618. Epub 2008 Jan 25.

8. Klein R, Klein BE, Magli YL, Brothers RJ, Meuer SM, Moss SE, Davis MD. An alternative method of grading diabetic retinopathy. Ophthalmology 1986 Sep; 93(9):1183-7.

9. Agarwal S, Raman R, Paul PG, Rani PK, Uthra S, Gayathree R, McCarty C, Kumaramanickavel G, Sharma T. Sankara Nethralaya-Diabetic Retinopathy Epidemiology and Molecular Genetic Study (SNDREAMS 1): study design and research methodology. Ophthalmic Epidemiol. 2005 Apr;12(2):143-53. 
10. Tapp RJ, Tikellis G, Wong TY, Harper CA, Zimmet PZ, Shaw JE; Australian Diabetes Obesity and Lifestyle Study Group. Longitudinal association of glucose metabolism with retinopathy: results from the Australian Diabetes Obesity and Lifestyle (AusDiab) study. Diabetes Care. 2008 Jul; 31(7):1349-54. doi: 10.2337/dc07-1707. Epub 2008 Apr 14.

11. Brown JB, Pedula KL, Summers KH. Diabetic retinopathy: contemporary prevalence in a wellcontrolled population. Diabetes Care. 2003 Sep; 26(9): 2637-42.

12. Anitha B, Sampathkumar R, Balasubramanyam M, Rema M. Advanced glycation index and its association with severity of diabetic retinopathy in type 2 diabetic subjects. J Diabetes Complications. 2008 Jul-Aug;22 (4):261-6. doi: 10.1016/j.jdiacomp.2007.05.005. Epub 2008 Apr 16.

13. Schorr SG, Hammes HP, Müller UA, Abholz HH, Landgraf R, Bertram B. The Prevention and Treatment of Retinal Complications in Diabetes. Dtsch Arztebl Int. 2016 Dec 2;113(48):816-823. doi: 10.3238/arztebl. 2016.0816 .

14. Liew G, Wong VW, Ho IV. Mini-Review: Changes in the Incidence of and Progression to Proliferative and
Sight-Threatening Diabetic Retinopathy Over the Last 30 Years. Ophthalmic Epidemiol. 2017 Jan 19: 1-8.doi: 10.1080/09286586.2016.1259638.

15. Akil H, Buluş AD, Andiran N, Alp MN. Ocular manifestations of Type 1 diabetes mellitus in pediatric population. Indian J Ophthalmol. 2016 Sep;64(9):654658. doi: 10.4103/0301-4738.194336.

16. Roy S, Kern TS, Song B, Stuebe C. Mechanistic Insights into Pathological Changes in the Diabetic Retina: Implications for Targetng Diabetic Retinopathy. Am J Pathol. 2017 Jan; 187(1): 9-19. doi: 10.1016/j. ajpath.2016.08.022.

17. Amin ZA, Islam QU, Mehboob MA. Comparison of serum lipid profile in Type-2 Diabetics with and without retinopathy in Pakistani population. Pak J Med Sci. 2016 Nov-Dec;32(6):1349-1353. doi: 10. 12669 /pjms. 326.11056.

18. Umayahara Y, Fujita Y, Watanabe H, Kasai N, Fujiki N, Hatazaki M, Koga M. Association of glycated albumin to HbA1c ratio with diabetic retinopathy but not diabetic nephropathy in patients with type 2 diabetes. Clin Biochem. 2016; Dec 5. pii: S0009-9120 (16)30390-3. doi: 10.1016/j.clinbiochem. 2016.11.032.

\section{How to cite this article?}

Priyadharshini N, Annamalai R, Muthayya M.K. The relationship between blood sugar levels (glycosylated haemoglobin) and the risk of development of diabetic retinopathy. Int J Med Res Rev 2017;5(01):61-67. doi:10.17511/ijmrr. 2017.i01.09. 